\title{
Integrating metalloporphycenes into p-type NiO-based dye-sensitized solar cells
}

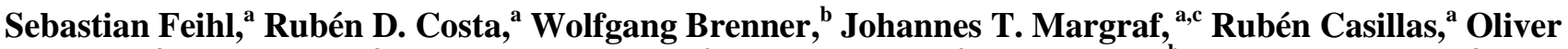 \\ ${ }_{5}$ Langmar, ${ }^{\mathrm{a}}$ Anne Browa, ${ }^{\mathrm{a}}$ Tatyana E. Shubina, ${ }^{\mathrm{c}}$ Timothy Clark, ${ }^{\mathrm{c}}$ Norbert Jux, ${ }^{\mathrm{b}}$ and Dirk M. Guldi ${ }^{\mathrm{a}}$
}

Received (in XXX, XXX) Xth XXXXXXXXX 200X, Accepted Xth XXXXXXXXX 200X

First published on the web Xth $X X X X X X X X X 200 X$

DOI: $10.1039 / \mathrm{b} 000000 \mathrm{x}$

In the current work, we have explored a novel synthetic route ${ }_{10}$ towards metalated porphycenes and their use in p-type NiObased dye-sensitized solar cells. Particular emphasis is placed on the influence that the relative positioning of the anchoring group exerts on the DSSC performance.

Factors that favor dye-sensitized solar cells (DSSCs) as a 15 key technology in solar-energy conversion schemes include low purity requirements and easy accessibility, low costs, fast processing, and simple up-scaling. DSSCs have been rendered even more attractive by recent progress in solid-state devices and substrate flexibility. ${ }^{1,2}$ To date, record efficiencies as 20 high as $13 \%$ have been reported for n-type DSSCs. ${ }^{3}$ However, efficient p-type DSSCs, which complement n-type DSSCs in, for example, tandem configurations, are necessary for obtaining values beyond $13 \%$, and are therefore the subject of intensive investigation. ${ }^{4}$

25 Regardless of whether the semiconductor materials are nor p-type, the chromophore attached to the semiconductor electrode is decisive in terms of light harvesting, charge transfer, and charge transport. Very diverse organic or inorganic chromophores have recently been tested in both 30 types of DSSCs. ${ }^{5-8}$ Fine tuning synthetic parameters such as the metalation of the chromophore, the nature of the anchor, and the bridge that links the chromophore to the anchor, have made control over important characteristics like highly effective charge separation, reduced charge recombination, 35 and long term stability possible. ${ }^{9-12}$

Semiconductor nickel oxide (NiO) stands out as an electrode material for p-type DSSCs. ${ }^{7,13-15}$ This has triggered the design of novel light-harvesting and electron-accepting chromophores. ${ }^{13} \mathrm{We}$ have focused on porphycenes because of 40 their similarity to porphyrins and phthalocyanines. ${ }^{16,} 17$ In contrast to the electron-donating features of porphyrins and phthalocyanines, which are widely used in n-type DSSCs, ${ }^{8,18}$ porphycenes are strong electron acceptors, making them more suitable building blocks for p-type DSSCs. To the best of our

45 knowledge, only a few examples of the use of free-base porphycenes in solar energy devices are known to date. ${ }^{19,20}$

We now report two major achievements. The first is the development of a new synthetic route to nickel porphycenes, which have barely been investigated previously. We have 50 characterized the novel nickel porphycenes physicochemical and computational techniques. Secondly, we have used these nickel porphycenes in p-type NiO-based DSSCs. We have been able to rationalize the overall performance of the resulting devices in terms of the position and the nature of the 55 anchor groups and, in turn, to establish new guidelines to designing novel electron acceptors.

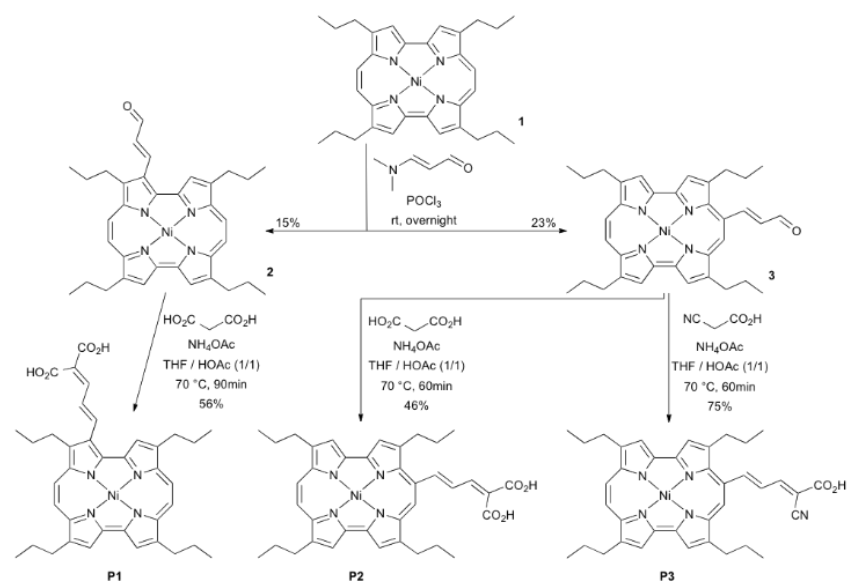

Scheme 1. Synthetic routes towards porphycenes P1-P3.

Three different nickel porphycenes were prepared ${ }_{60}$ Scheme 1. Experimental details are given in the supporting information (SI). In order to obtain the target porphycenes, the 2,7,12,17-tetra-n-propylporphycenato nickel (1) was chosen as starting material. Firstly, the peripheral alkyl chains ensure sufficient solubility in a wide variety of solvents. Secondly, ${ }_{65}$ the well-established chemistry of $\mathbf{1}$ allows preparing the target featuring a vinyl linker, which has been established as an efficient linker for porphyrins in n-type DSSCs. ${ }^{21}$ Thirdly, we decided to test the viability of two different anchoring groups, that is, dicarboxyl and cyanocarboxy groups. Finally, we 70 focused only on nickel porphycene to avoid metalations when using free-base derivatives.

In detail, $\mathbf{1}$ is available in good yields through a wellknown eight-step synthesis ${ }^{16}$ and was reacted with 3dimethylaminoacrolein under Vilsmeier conditions to obtain 75 formylvinyl-functionalized porphycenes in accordance with a previously published procedure. ${ }^{22}$ The two isomers obtained were separated by repeated column chromatography. 2 was then reacted in a Knoevenagel reaction with malonic acid in the presence of ammonium acetate, which led to dicarboxylic 80 acid $\mathbf{P 1}$ in $56 \%$ yield after purification by column chromatography. Similarly, aldehyde $\mathbf{3}$ was reacted to give the corresponding $\mathbf{P 2}$ in $46 \%$. P3, which bears a cyanoacetic acid 
anchoring group, was generated from $\mathbf{3}$ in $75 \%$ yield as a complement to $\mathbf{P 2}$.

Absorption and fluorescence spectroscopy and electrochemical assays provide insight into the electronic 5 properties of the porphycenes. The absorption spectra of P1, P2, and P3 feature Soret and Q-band absorptions at 400 and $600 \mathrm{~nm}$, respectively (see Figure 1 and Table S1). As for porphyrins and phthalocyanines, ${ }^{17}, 23,24$ comparing the absorption spectra of P1, P2, and P3 to that of the free-base 10 porphycene discloses the impact of the metalation and of the anchor substituent. ${ }^{16,25}$

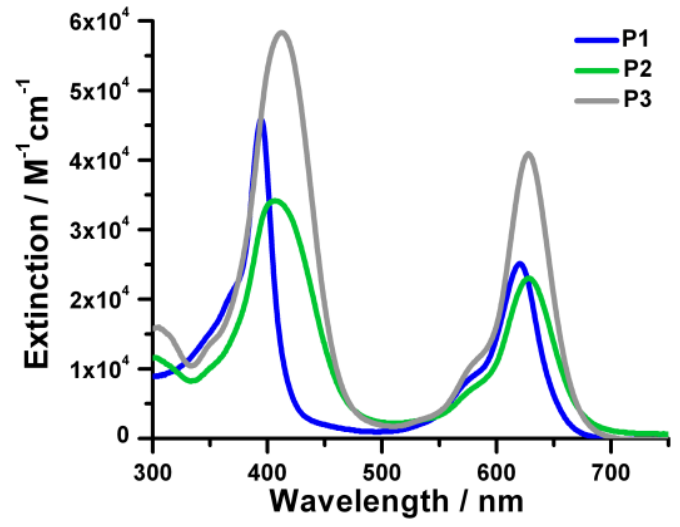

Figure 1 Steady-state absorption spectra of P1, P2, and P3 in DMF.

15 Metalation causes bathochromic shifts of the absorption maxima from $370 \mathrm{~nm}$ to 395,407 , and $412 \mathrm{~nm}$ for P1, P2, and P3, respectively. ${ }^{17,24}$ The linker substituents lower the overall symmetry from $D_{2 h}$ to $C_{s}$, ${ }^{16}$ resulting in broadening of the Soret band absorption in the 350 to $500 \mathrm{~nm}$ range. ${ }^{17,} 23$ ${ }_{20}$ Significantly red-shifted and broadened Soret and Q-band absorptions are found on changing the position of the anchor substituent from, for example, the pyrrole-rings in P1 to the ethylene bridges in $\mathbf{P 2}$ and $\mathbf{P 3}{ }^{17}$ In $\mathbf{P 3}$, the presence of electron-withdrawing cyano-groups further red-shifts the ${ }_{25}$ Soret band absorptions due to coplanarity ${ }^{17,}{ }^{26}$ Fluorescence was not observed for any of the porphycenes because of fast non-radiative deactivation processes, which are well known for $\mathrm{Ni}^{2+}$-based porphyrins. ${ }^{27,28}$

P1, P2, and P3 exhibit similar redox characteristics; three 30 quasi-reversible reductions around -0.80/-1.09/-1.56, -0.88/$1.16 /-1.61$, and $-0.99 /-1.27 /-1.78 \mathrm{~V}$ and two quasi-reversible oxidations around $0.98 / 1.31,1.02 / 1.22$ and $0.89 / 1.05 \mathrm{~V}$ versus NHE (see Figure S4). Porphycenes are slightly easier (1.02 V) to reduce and slightly more difficult $(-0.80 \mathrm{~V})$ to oxidize than 35 free-base and nickel porphyrins, confirming their electron accepting character (Table S2). ${ }^{29-31}$ Figure S5 shows the reduction and oxidation potentials of $\mathbf{P 1}, \mathbf{P 2}$, and $\mathbf{P 3}$, the valence band ( $0.54 \mathrm{~V}$ vs. $\mathrm{NHE})$ of $\mathrm{NiO}$, and the redox potential $\left(0.44 \mathrm{~V}\right.$ vs. NHE) of the $\mathrm{I}_{3}{ }^{-} / \mathrm{I}^{-}$couple. ${ }^{32}$ Taking these 40 into account, we hypothesize that these novel acceptors are potentially good sensitizers in NiO-based DSSCs.

To verify our hypothesis, we fabricated p-type DSSCs based on $\mathrm{NiO}$ electrodes by following the route reported by Sumikura et. al. ${ }^{33}$ Detailed information is provided in the SI.
$45 \mathrm{Next}$, the $\mathrm{NiO}$ electrodes were immersed in $10^{-4}$ molar DMF solutions of P1, P2, and P3. Adsorption kinetic experiments, as documented in Figure S9, confirm that the optimum uptake for realizing full electrode coverage is $90 \mathrm{~min}$ regardless of the linker and the anchoring group. It is important to point out 50 two aspects. Firstly, the light-harvesting efficiency of the electrodes is similar for all the porphycene electrodes (Figure S9), ensuring a reasonable comparison between the corresponding devices. Secondly, all the porphycenes reveal good photostability once attached onto the electrodes under 55 both room light and 1sun / AM 1.5 illumination conditions Figure S11.

Finally, the p-type DSSCs were completed with a platinum counter-electrode and $\mathrm{LiI} / \mathrm{I}_{2}(5: 1)$ in acetonitrile as electrolyte. The device performance is summarized in Table S3 and the 60 photocurrent density versus applied voltage graphs are shown in Figure 2.

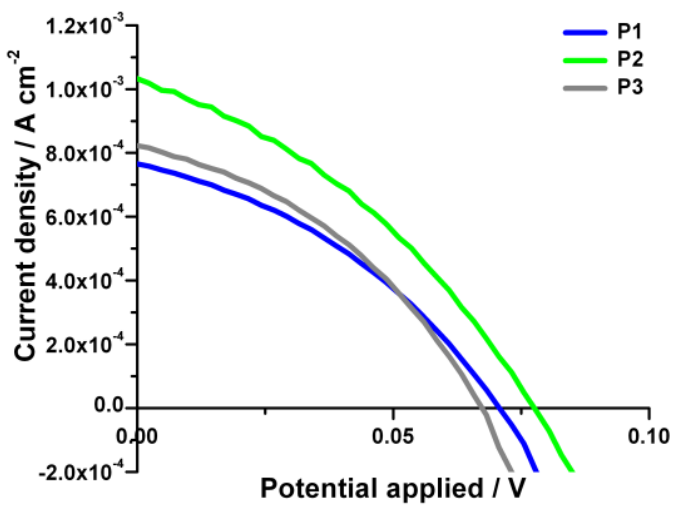

Figure 2 J-V-curves of p-type NiO-based DSSCs with P1, P2, and P3.

65 Several trends are discernable on close inspection of the device features. Firstly, the photocurrent densities for devices made with porphycenes that feature anchors at the ethylene bridges, $\mathbf{P 2}\left(1.03 \mathrm{~mA} \cdot \mathrm{cm}^{-2}\right)$ and $\mathbf{P 3}\left(0.82 \mathrm{~mA} \cdot \mathrm{cm}^{-2}\right)$, are higher than those for those prepared with $\mathbf{P 1}\left(0.77 \mathrm{~mA} \cdot \mathrm{cm}^{-2}\right)$, in 70 which the anchor is attached at the pyrrole ring. The latter trend is further corroborated by the incident photon-to-current efficiency (IPCE) spectra. For example, devices with P2 feature the highest Q-band contribution centered at $630 \mathrm{~nm}$. The featureless IPCE spectrum of bare NiO-based devices in 75 the region from 500-750 nm clearly confirms the role of the dyes as sensitizers. Please note that the Soret contribution overlaps with the electrolyte band, hampering any quantitative analysis - Figure S10. Next, among all of the porphycene devices, those with $\mathbf{P 2}$ feature the highest open-circuit voltage ${ }_{80}\left(\mathrm{~V}_{\mathrm{oc}}\right)$ and short-circuit current density $\left(\mathrm{J}_{\mathrm{sc}}\right)$ of $0.078 \mathrm{~V}$ and $1.03 \mathrm{~mA} / \mathrm{cm}^{2}$, respectively. Therefore, the highest efficiency $(0.028 \%)$ was found for $\mathbf{P 2}$ devices, followed by efficiencies of 0.021 and $0.019 \%$ for devices with P3 and P1, respectively. In contrast to the photostability of the 85 porphycenes adsorbed onto $\mathrm{NiO}$ electrodes, DSSCs show low stability. For example, the efficiency increases by around $30 \%$ relative to the initial value followed by a fast decay until $50 \%$ of the maximum efficiency value at $55 \mathrm{~h}$. Therefore, we conclude that our devices are not fully optimized in terms of 
electrolyte composition. As a confirmation, $\mathbf{J}_{\mathrm{sc}}$ versus illumination intensity plots show non-linear trends, indicating a poor balance between regeneration/recombination processes and/or diffusion problems in the electrolyte - Figure S12. ${ }^{34}$ ${ }_{5}$ Nevertheless, our results will be useful towards the design of new porphycenes for highly efficient p-type DSSCs, ${ }^{35}$ although the performances of our devices are still on a quite moderate level. ${ }^{7,36-38}$

In this context, we infer from Figure S5 that similar 10 energetics in any of the combinations of $\mathbf{P 1}, \mathbf{P 2}$, and $\mathbf{P 3}$ with $\mathrm{NiO}$, and $\mathrm{I}_{3}{ }^{-} / \mathrm{I}^{-}$should lead to constant results, at odds with the experimental results. Only the position and the nature of the anchor remain as the cause of the differences. For instance, the most effective scenario for hole-transfer to the valence 15 band of $\mathrm{NiO}$ is apparently found if the anchors are placed at the ethylene bridges (P2 and P3). The electron-withdrawing cyano-groups found in $\mathbf{P 3}$, however, retard the hole-transfer and reduce the photocurrent density and the overall device efficiency.
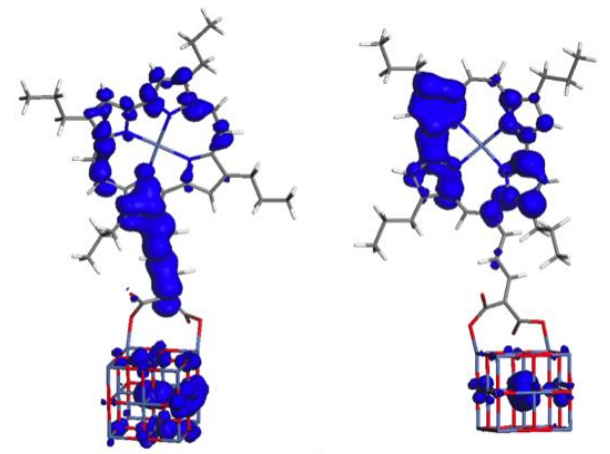

${ }_{20}$ Figure 3 Orbitals into which an electron is promoted in the lowest excited singlet state $\left(S_{1}\right)$ for $\mathbf{P 1}$ (left) and P2 (right).

To support these ideas, semiempirical (PM6) ${ }^{39}$ unrestricted natural orbital pair excitation configuration interaction (UNOPECI $)^{40}$ calculations were performed to shed light on the 25 interfacial charge injection processes. The systems calculated consisted of $\mathbf{P 1}$ and $\mathbf{P 2}$ linked to an $\mathrm{Ni}_{14} \mathrm{O}_{13}{ }^{2+}$ cluster. The molecular structures of $\mathbf{P 1}$ and $\mathbf{P 2}$ and the linker/NiO hybrid were optimized, and then joined for the PECI calculations. This guaranteed that the results were not influenced by 30 changes in the binding motif. Next, we analyzed the electronic structure of the lowest-energy excited state of both systems. The charge redistribution upon excitation can be quantified by the summed Coulson atomic charges of dye, bridge, and $\mathrm{NiO}$ in the ground and excited states (see Table S4). This reveals 35 that the electronic coupling between $\mathbf{P 2}$ and $\mathrm{NiO}$ displays a partial transfer of electrons from $\mathrm{NiO}$ to the porphycene. For $\mathbf{P 1}$, the $S_{1}$ state is, in stark contrast, characterized by a net electron transfer to $\mathrm{NiO}$ and to the bridge. Such a scenario favors the unwanted charge-recombination processes. The 40 difference between the $S_{1}$ states of the two dyes can be visualized by plotting the orbitals into which the electron is promoted (Fig. 3).

Finally, electrochemical impedance spectroscopy (EIS) measurements were performed (details are given in the SI).

${ }_{45}$ The Nyquist and Bode phase plots are shown in Figures S14 and $\mathrm{S} 15$, respectively. In addition, the resistance to charge transfer processes (R), and the capacitance (C) are summarized in Tables S5 and S6. For p-type DSSCs, EIS provides insight into charge-transfer processes across the 50 electrode/chromophore and the electrode/electrolyte interfaces with and without illumination, respectively. Under illumination and forward bias, the rate of charge transfer between the electrode and porphycenes should be high and $R_{i}$ should be low. On the contrary, under dark conditions and ${ }_{55}$ forward bias, the rate of charge transfer between the electrolyte and electrode should be low and $R_{d}$ should be high. $^{41,42}$ Our observations that the highest photocurrent densities are found for $\mathbf{P 2}$ devices are consistent with the above-mentioned guidelines, having the lowest $\mathrm{R}_{\mathrm{i}}$ and the 60 highest $R_{d}$ among all of the tested porphycenes (see Tables S5 and S6). In addition, the rate constant $\left(k_{\mathrm{eff}}\right)$ for electrode/chromophore charge transfer is the highest for P2 devices. In line with the theoretical characterization, P1 devices exhibit the highest $R_{i}$ and, in turn, the least efficient ${ }_{65}$ electron transfer process under operating conditions.

In summary, we document for the first time the synthesis of novel nickel porphycenes, their photophysical and electrochemical features, as well as their application in p-type DSSCs. Molecular-orbital calculations and impedance 70 measurements helped establish a comprehensive understanding of the bottlenecks in the overall device performance. Still, transient absorption spectroscopy and small-angle-X-ray-scattering surface characterization assays are necessary to shed light onto the kinetics of injection,

75 recombination, and regeneration processes as well as the reorganization of the molecules onto the electrode surface. Despite the moderate performances, we believe that future progress in the synthesis will help to establish nickel porphycenes as a new kind of sensitizers for p-type DSSCs. 80 Ongoing work in our laboratory is focused on the aforementioned aspects.

Acknowledgements. The authors thank the German Science Council (DFG) for the financial support in the framework the Cluster of Engineering of Advanced Materials (EAM) and the ${ }_{85}$ Bavarian State Government as part of the Solar technologies goes Hybrid initiative. JM thanks the Beilstein Institute for the award of a fellowship.

\section{Notes and references}

${ }^{a}$ Friedrich-Alexander-University Erlangen-Nuremberg, 90 Department of Chemistry and Pharmacy \& Interdisciplinary Center for Molecular Materials, Egerlandstr. 3, 91058 Erlangen, Germany.E-mail: dirk.guldi@fau.de. ${ }^{b}$ Friedrich-AlexanderUniversity Erlangen-Nuremberg, Department of Chemistry and Pharmacy, Henkestr. 42, 91054 Erlangen, Germany. ${ }^{c}$ Friedrich${ }_{95}$ Alexander-University Erlangen-Nuremberg, Department of Chemistry and Pharmacy, Computer Chemistry Center, Nägelsbachstr. 25, 91052 Erlangen, Germany.

1. H. M. Upadhyaya, S. Senthilarasu, M.-H. Hsu and D. K. $100 \quad$ Kumar, Sol. Energy Mater. Sol. Cells, 2013, 119, 291.

2. H.-P. Kuo and C.-T. Wu, Sol. Energy Mater. Sol. Cells, 2014, 120, 81 .

3. A. Yella, H.-W. Lee, H. N. Tsao, C. Yi, A. K. Chandiran, M. K. Nazeeruddin, E. W.-G. Diau, C.-Y. Yeh, S. M. Zakeeruddin and M. Grätzel, Science, 2011, 334, 629. 

4. A. Nattestad, A. J. Mozer, M. K. Fischer, Y. B. Cheng, A. Mishra, P. Bauerle and U. Bach, Nat Mater, 2010, 9, 31.
5. E. Yoneda, M. K. Nazeeruddin and M. Craetzel, $J$. Photopolym. Sci. Technol., 2012, 25, 175.
$56 . \quad$ L.-Y. Lin, C.-H. Tsai, K.-T. Wong, T.-W. Huang, C.-C. Wu, S.-H. Chou, F. Lin, S.-H. Chen and A.-I. Tsai, J. Mater. Chem., 2011, 21, 5950.
$7 . \quad$ L. Le Pleux, A. L. Smeigh, E. Gibson, Y. Pellegrin, E. Blart, G. Boschloo, A. Hagfeldt, L. Hammarstrom and F. Odobel,
8. M. V. Martinez-Diaz, G. de la Torre and T. Torres, Chem. Commun., 2010, 46, 7090.
9. M. Garcia-Iglesias, J.-H. Yum, R. Humphry-Baker, S. M. Zakeeruddin, P. Pechy, P. Vazquez, E. Palomares, M. Gratzel,
15
$10 . \quad$ Z. Ji, G. Natu, Z. Huang and Y. Wu, Energy Environ. Sci., 2011, 4, 2818.
11. P. Qin, H. Zhu, T. Edvinsson, G. Boschloo, A. Hagfeldt and L. Sun, J. Am. Chem. Soc., 2008, 130, 8570.
$2012 . \quad$ N. Robertson, Angew. Chem. Int. Ed., 2006, 45, 2338.
13. F. Odobel and Y. Pellegrin, J. Phys. Chem. Lett., 2013, 4, 2551.

14. S. Feihl, R. D. Costa, S. Pflock, C. Schmidt, J. Schonamsgruber, S. Backes, A. Hirsch and D. M. Guldi, RSC

25

15. E. A. Gibson, L. Le Pleux, J. Fortage, Y. Pellegrin, E. Blart, F. Odobel, A. Hagfeldt and G. Boschloo, Langmuir, 2012, 28, 6485.

16. E. Vogel, M. Balci, K. Pramod, P. Koch, J. Lex and O. Ermer, 30

17. L. Jiang, R. A. Zaenglein, J. T. Engle, C. Mittal, C. S. Hartley, C. J. Ziegler and H. Wang, Chem. Commun., 2012, 48, 6927.

18. I. López-Duarte, M. Wang, R. Humphry-Baker, M. Ince, M. V. Martínez-Díaz, M. K. Nazeeruddin, T. Torres and M. Grätzel, Angew. Chem. Int. Ed., 2012, 51, 1895.

19. W. Brenner, J. Malig, R. D. Costa, D. M. Guldi and N. Jux, Adv. Mater., 2013, 25, 2314.

$20 . \quad$ R. D. Costa, J. Malig, W. Brenner, N. Jux and D. M. Guldi, Adv. Mater., 2013, 25, 2600.

$4021 . \quad$ W. M. Campbell, K. W. Jolley, P. Wagner, K. Wagner, P. J. Walsh, K. C. Gordon, L. Schmidt-Mende, M. K. Nazeeruddin, Q. Wang, M. Grätzel and D. L. Officer, J. Phys. Chem. C, 2007, 111, 11760 .

22.

33. S. Sumikura, S. Mori, S. Shimizu, H. Usami and E. Suzuki, $J$. Photochem. Photobiol. A, 2008, 199, 1

34. S. Y. Huang, G. Schlichthörl, A. J. Nozik, M. Grätzel and A. J. Frank, J. Phys. Chem. B, 1997, 101, 2576.

70 35. It is important to point out that, although the device efficiencies are lower than those of the state-of-the-art, ${ }^{76-38}$ the presented trends are obtained after analyzing several devices (see SI). Thus, these devices had in common the same
75 36. absorption features, electrode thickness, and electrolyte composition.

S. Powar, T. Daeneke, M. T. Ma, D. Fu, N. W. Duffy, G. Götz, M. Weidelener, A. Mishra, P. Bäuerle, L. Spiccia and U. Bach, Angew. Chem Int. Ed., 2013, 52, 602.

S. Powar, Q. Wu, M. Weidelener, A. Nattestad, Z. Hu, A. Mishra, P. Bauerle, L. Spiccia, Y.-B. Cheng and U. Bach, Energy Environ. Sci., 2012, 5, 8896.

X. L. Zhang, F. Huang, A. Nattestad, K. Wang, D. Fu, A. Mishra, P. Bauerle, U. Bach and Y.-B. Cheng, Chem. Commun., 2011, 47, 4808.

J. P. Stewart, J Mol Model, 2007, 13, 1173.

P. O. Dral and T. Clark, J. Phys. Chem. A, 2011, 115, 11303.

Z. Huang, G. Natu, Z. Ji, M. He, M. Yu and Y. Wu, J. Phys. Chemi. C, 2012, 116, 26239.

C, 2011, 115, 25109 . 